\title{
Classical simulations and particle production in heavy-ion collisions
}

\author{
Jon-Ivar Skullerud ${ }^{\mathrm{a}}$

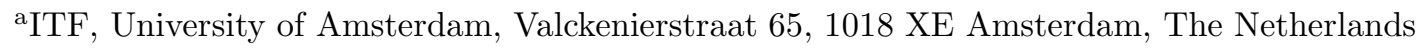

The classical approximation may be applied to a number of problems in non-equilibrium field theory. The principles and limits of classical real-time lattice simulations are presented, with particular emphasis on the definition of particle numbers and energies and on applications to the earliest stages of heavy-ion collisions.

\section{INTRODUCTION}

One of the most important predictions of lattice QCD is the existence of a transition from ordinary hadronic matter, where quarks and gluons are confined, to a quark-gluon plasma at high temperatures. Current estimates are that this transition takes place at $T_{c} \sim 150-170 \mathrm{MeV}$, and is most likely a crossover at zero chemical potential. At higher chemical potential a firstorder phase transition is predicted, ending in a tricritical point at $\mu_{B}^{E} \sim 100-500 \mathrm{MeV}$.

These predictions are currently being put to the test at RHIC and other heavy-ion colliders. However, interpreting the results of these experiments and comparing them to lattice QCD predictions is far from straightforward. One important reason for this is that the process to a large extent takes place out of thermal equilibrium, and equilibrium field theory methods such as lattice Monte Carlo are therefore not sufficient.

One major, still unsolved puzzle of heavyion physics is whether the system ever actually reaches thermal equilibrium, and if so, what the equilibration time is. Many aspects of the collision can be successfully described (eg, using hydrodynamics [1]) by assuming a very short equilibration time of $1 \mathrm{fm} / c$, but it is far from understood how this would come about. Clearly, a proper understanding of the thermalisation process is essential if we are to have a coherent and reliable description of the heavy-ion collision.

At late stages of the collision process, as the system expands, the particles eventually decouple. This typically occurs in two phases: first, inelastic collisions cease, causing the ratios of dif- ferent particle species to be fixed (chemical freezeout); later, also the mean free path for elastic collisions becomes too large and the momentum distribution of the particles deviates from that of thermal equilibrium (thermal or kinetic freezeout). It is at this point that the final particle yields of the collision are fixed. Thus, nonequilibrium dynamics is needed to understand also this aspect of the process.

Another field where non-equilibrium field theory is needed, is early-universe physics. Examples of non-equilibrium processes which require a fieldtheoretical approach include (p)reheating, electroweak and QCD phase transitions in the early universe, and baryogenesis.

Field theories out of equilibrium is a notoriously difficult problem to study nonperturbatively. A large number of approaches have been employed, including Hartree and large-N approximations [23], Dyson-Schwinger-related approaches based on the 2PI effective action [45], and kinetic theory [6]. All of these have their strengths as well as drawbacks and limitations. Within its area of applicability, the classical approximation has the advantage of being fully nonperturbative, easy and relatively inexpensive to implement numerically, and straightforwardly applicable to gauge theories. The major drawback is obviously that quantum effects are not taken into account.

\section{THE CLASSICAL APPROXIMATION}

Classical statistical physics is quantum statistical physics in the limit of large occupation numbers. It follows that the classical approximation 
can be used when the occupation numbers of the relevant or dominant modes of the system are large. In the context of heavy-ion collisions it is arguable that the multiplicity of soft gluons in the initial (pre-equilibrium) stages is very high, and the classical approximation may therefore be valid.

The classical approximation has also been applied to the chiral dynamics during freeze-out. In the linear sigma model and related models, the effective potential of the chiral $(\sigma, \vec{\pi})$ field changes from symmetric to Mexican-hat type as the temperature drops, giving rise to an instability where the low-momentum modes increase exponentially. In this scenario, the resulting high occupation numbers justify the use of the classical approximation.

A particular hazard with simulating classical dynamics on a lattice is connected with highmomentum lattice artefacts. These will in general interact with the soft modes which carry the interesting physics and for which the classical approximation is in principle valid. If there is sufficient strength in the hard modes, they may equilibrate classically with the soft modes on a much shorter timescale than that of the interesting physics 7 . In that case, not only the hard modes, but the entire system will be dominated by classical lattice artefacts.

To avoid this problem, it is important that the high-momentum modes should be, and remain, strongly suppressed. At early stages, this can be ensured by choosing appropriate initial conditions.

The initial conditions are a crucial part of the simulation. They should reflect the salient features of the system at the outset. This is also the only place where information about the quantum nature of the real world enters into the simulation. A quantum system may be represented as an ensemble of classical configurations initially distributed according to quantum statistics [8]. One example of this may be to choose the 2-point correlators of the fields and their canonical momenta to obey the Bose-Einstein distribution for free fields at some temperature $T$, after subtracting the quantum vacuum fluctuations. For scalar fields $\phi$ with momentum fields $\pi$ and mass $m$ one would then have

$$
\begin{aligned}
\langle\phi(\vec{k}, t=0) \phi(-\vec{k}, 0)\rangle & =\frac{1}{\omega_{k}} \frac{1}{e^{\omega_{k} / T}-1}, \\
\langle\pi(\vec{k}, 0) \pi(-\vec{k}, 0)\rangle & =\omega_{k} \frac{1}{e^{\omega_{k} / T}-1}
\end{aligned}
$$

where $\omega_{k}=\sqrt{\vec{k}^{2}+m^{2}}$. Such an initialisation also provides an exponential cutoff for the hard modes, which will help in avoiding the dangerous lattice artefacts. Following this, each configuration evolves independently according to the classical hamiltonian equations of motion, and timedependent correlators are computed as averages over the initial conditions.

Given sufficient time, the system will eventually thermalise classically, resulting in classical equipartition $n_{k}=\omega_{k} / T$ and giving rise to Rayleigh-Jeans type divergences. The hope is that this will happen on much longer time scales than those under consideration in the simulation.

\section{DISTRIBUTION FUNCTIONS}

There is no unique definition of local particle numbers and energies for interacting fields out of equilibrium. Still, the system may exhibit effective particle-like behaviour, which may be used to characterise the approach to thermal equilibrium or to an equilibrium-like distribution. Given a definition of local particle numbers, these can also be used to give an effective description of the system in terms of kinetic theory.

The effective particle numbers may be extracted from the two-point field correlators, which in the free-field case (where the particle description is appropriate and well-defined) contain all the information there is about the system. For example, for a homogeneous, free scalar field we have

$$
\begin{aligned}
& \langle\phi(\vec{k}, t) \phi(-\vec{k}, t)\rangle=\frac{1}{\omega_{k}}\left(n_{k}+\frac{1}{2}\right) \\
& \langle\pi(\vec{k}, t) \pi(-\vec{k}, t)\rangle=\omega_{k}\left(n_{k}+\frac{1}{2}\right)
\end{aligned}
$$

where $n_{k}$ is the occupation number for the mode with momentum $\vec{k}$ and $\omega_{k}$ is the associated energy. For interacting fields, this may in turn be 
used as a definition of the instantaneous particle numbers and energies $n$ and $\omega$ 98:

$$
\begin{aligned}
n_{k}(t)+\frac{1}{2} & \equiv \sqrt{\langle\phi(\vec{k}, t) \phi(-\vec{k}, t)\rangle\langle\pi(\vec{k}, t) \pi(-\vec{k}, t)\rangle} \\
\omega_{k}(t) & \equiv \sqrt{\frac{\langle\pi(\vec{k}, t) \pi(-\vec{k}, t)\rangle}{\langle\phi(\vec{k}, t) \phi(-\vec{k}, t)\rangle}}
\end{aligned}
$$

In the classical approximation the $1 / 2$ is left out.

In a non-abelian gauge theory, the correlation functions will in general be gauge dependent, so the distribution functions will contain ambiguities due to the gauge choice. This ambiguity may be removed by constructing gauge invariant correlators using parallel transporters; however, this introduces path dependence. In particular, in a lattice regularisation there is in general no one preferred path between two points.

Although the distribution functions are not unique, all physical observables extracted from them, such as masses, temperatures and chemical potentials, should not depend on the definition and in particular on the gauge. As long as these quantities are not well-defined on the other hand (such as when the system is very far from equilibrium and the quasiparticle picture does not apply), one may expect "masses" and "temperatures" to be definition-dependent. Thus, studying the gauge dependence (or path dependence) of distribution functions may serve the double purpose of monitoring the approach to equilibrium and verifying the validity of the approach used.

One natural choice of gauge is the Coulomb gauge, which is a smooth gauge. In a system with spontaneously broken gauge symmetry (e.g., a Higgs system), the unitary gauge, where the (effective) Higgs field has only one non-zero, real component, is another natural choice. Other gauges, such as maximal abelian gauge, axial gauges or random gauge, may also be considered.

In the Coulomb gauge, the gauge potential $A_{i}$ (but not its conjugate momentum $E_{i}$ ) is purely transverse, and it can be shown that the transverse free correlators behave analogously to the scalar case 10]. Thus the particle numbers and energies can be defined as

$$
n_{k} \equiv \sqrt{D_{T}^{A}(k) D_{T}^{E}(k)}, \quad \omega_{k} \equiv \sqrt{\frac{D_{T}^{E}(k)}{D_{T}^{A}(k)}} .
$$

Here $D_{T}^{A}, D_{T}^{E}$ are the transverse $A$ - and $E$-correlators respectively, constructed from the two-point functions $\left\langle\Phi_{i}^{a}(\vec{k}) \Phi_{j}^{b}(-\vec{k})\right\rangle=\delta^{a b} C_{i j}^{\Phi \Phi}(\vec{k}) ; \Phi=A, E$ according to

$$
\begin{aligned}
& C_{i j}^{A A}(\vec{k})=\left(\delta_{i j}-\frac{k_{i} k_{j}}{k^{2}}\right) D_{T}^{A}(k), \\
& C_{i j}^{E E}(\vec{k})=\left(\delta_{i j}-\frac{k_{i} k_{j}}{k^{2}}\right) D_{T}^{E}(k)+\frac{k_{i} k_{j}}{k^{2}} D_{L}^{E}(k) .
\end{aligned}
$$

In figure 11 the gauge dependence of effective particle numbers is illustrated in the $\mathrm{SU}(2)$-Higgs model [10]. In this case, the system was prepared in such a way that all the energy initially was in the Higgs field, while the gauge potential was initialised to zero. Since the angular modes of the Higgs fields are absorbed into the gauge fields in the unitary gauge, the inital occupation numbers are very different. However, already after $t=10 m_{H}^{-1}$ the two distributions appear almost identical. However, while the particle numbers in the Coulomb gauge change very little from here on, in the unitary gauge they continue to fluctuate and it is only from $t \approx 40 m_{H}^{-1}$ on that one with some confidence can claim the numbers are gauge independent. This agrees roughly with the point where the dispersion relation in the unitary gauge begins to show stable, particle-like behaviour in this particular model.

In a non-homogeneous system, and in general in a kinetic-theory description, it is appropriate to think in terms of local particle numbers $n(\vec{x}, \vec{k}, t)$. These may be related to the Wigner functions constructed from gauge invariant two-point functions 9], or more generally to the two-point functions fourier transformed on a region $R(\vec{x})$ centred on $\vec{x}$. In the case of a scalar theory we may have (suppressing the common $t$-coordinate for 
brevity)
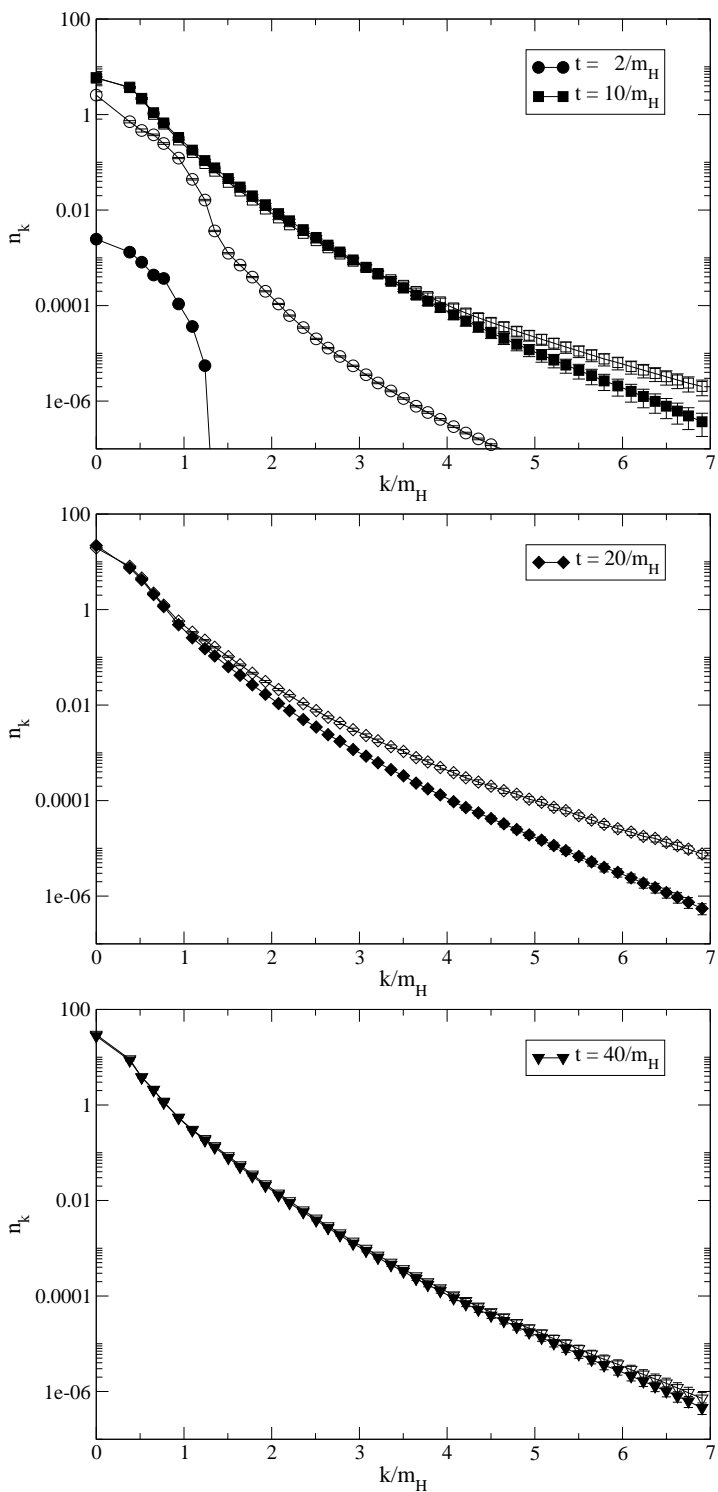

Figure 1. Gauge dependence of gauge particle numbers in the SU(2)-Higgs model [10] at different times. The filled symbols are particle numbers obtained in the Coulomb gauge, while the open symbols are for the transverse modes in the unitary gauge.

$$
\begin{aligned}
& C_{\phi \phi}(\vec{x}, \vec{k})=\frac{1}{\Omega_{R}} \int_{R(\vec{x})} d^{3} z d^{3} y e^{-i \vec{k} \cdot(\vec{y}-\vec{z})}\langle\phi(\vec{y}) \phi(\vec{z})\rangle, \\
& C_{\pi \pi}(\vec{x}, \vec{k})=\frac{1}{\Omega_{R}} \int_{R(\vec{x})} d^{3} z d^{3} y e^{-i \vec{k} \cdot(\vec{y}-\vec{z})}\langle\pi(\vec{y}) \pi(\vec{z})\rangle .
\end{aligned}
$$

Here $\Omega_{R}$ is the volume of the region $R(\vec{x})$. This coarse-graining creates an intrinsic unsharpness in the momentum $\vec{k}$ and position $\vec{x}$ of the quasiparticles, given by the size (and shape) of the region $R$.

If the system under consideration is homogeneous, we may improve statistics by performing an average over all space. This can be shown to be equivalent to local averaging in momentum space, with a weight function $w$ depending on the size and shape of $R$ :

$$
\begin{aligned}
C(\vec{k}, t) & =\frac{1}{V} \int d^{3} x C(\vec{x}, \vec{k}, t) \\
& =\frac{1}{V} \int d^{3} x C_{R(\vec{x})}(\vec{k}, t) \\
& =\sum_{\vec{p}} w(\vec{p}-\vec{k}) C_{V}(\vec{p}, t),
\end{aligned}
$$

where $C_{V}$ denotes the correlation function evaluated on the total volume $V$, and the sum is over the discrete momenta available on this volume. In practice, it is simpler to work backwards, choosing a simple form of momentum-space averaging which may correspond to rather complicated spatial regions. For instance, binning in the absolute value of the momentum,

$w(\vec{p}-\vec{k}) \propto \Theta\left(|\vec{k}|-|\vec{p}|+\frac{\Delta}{2}\right)-\Theta\left(|\vec{k}|-|\vec{p}|-\frac{\Delta}{2}\right)$,

corresponds to spherical shells with thickness approximately $1 / \Delta$ in position space.

\section{PURE YANG-MILLS}

At the earliest stages of heavy-ion collisions, the gluon density is expected to be so high that the classical approximation can be justified. The same approximation also justifies ignoring the 
back-reaction of the quarks, since their number density will be much lower; leaving us with classical Yang-Mills equations of motion, which may be solved numerically on a lattice.

The lattice equations of motion in the temporal gauge $\left(A_{0}=0\right)$ read

$\partial_{t} E_{i}^{a}(x)=D_{j}^{a b} \operatorname{tr}\left[i t^{b} U_{j i}(x)\right]$

where

$E_{i}^{a}(x)=F_{0 i}^{a}(x)=\operatorname{tr}\left[t^{a} U_{i}(x) U_{i}^{\dagger}(x+\hat{t})\right]$

is the canonical momentum to $A_{i}^{a}$. Here, $\partial_{t}$ denotes the backward lattice derivative, while $D_{j}$ is the backward covariant lattice derivative. The equations of motion for $A_{0}^{a}$ constitute the Gauss constraint,

$D_{i}^{a b} E_{i}^{b}=0$,

which must be satisfied by the initial conditions but is conserved by the equations of motion.

The initial gluon fields should be related to the gluon distributions of the two colliding nuclei: in principle they should just be the superposition of two Lorentz-boosted nuclear gluon distributions. Simulations have been carried out over a number of years by Krasnitz, Nara and Venugopalan 1112 (see also 13]) using the "colour glass condensate" model of the nuclear wave function to provide the initial conditions. In these studies, the numerical work has been simplified by considering only the mid-rapidity region where the physics is assumed to be boost-invariant. This reduces the system to effectively $2+1$ dimensions. With these assumptions, the authors have been able to provide an estimate of the initial energy density and gluon distribution which may be used as input into hydrodynamic or kinetic calculations.

An alternative approach would be to determine the nuclear gluon field from e.g. a bag model, give this a Lorentz boost, and perform a $2+1+1$ dimensional simulation with the longitudinal lattice spacing $a_{z}=a_{\perp} / \gamma$, where $a_{\perp}$ is the lattice spacing in the transverse $(x, y)$ direction. Work is underway to implement this.

\section{SUMMARY}

The classical approximation may be applied to a range of problems in non-equilibrium field theory where occupation numbers are high, such as the earliest stages of heavy-ion collisions. It has the advantage of being non-perturbative and computationally relatively inexpensive. Effective particle numbers may be defined out of equilibrium in a self-consistent manner, and their gauge dependence (or that of derived quantities such as masses and temperatures) can be used as a check on the validity of the quasi-particle picture.

\section{Acknowledgments}

This work was supported by FOM/NWO. I am thankful to Jan Smit and Anders Tranberg for numerous fruitful discussions.

\section{REFERENCES}

1. P. F. Kolb and U. Heinz, nucl-th/0305084

2. F. Cooper et al., Phys. Rev. D50, 2848 (1994) hep-ph/9405352.

3. D. Boyanovsky, H. J. de Vega and R. Holman, Phys. Rev. D51, 734 (1995) hep-ph/9401308.

4. J. Berges and J. Cox, Phys. Lett. B517, 369 (2001) hep-ph/0006160.

5. J. Berges and J. Serreau, hep-ph/0302210

6. R. Baier, A. H. Mueller, D. Schiff and D. T. Son, Phys. Lett. B502, 51 (2001) hep-ph/0009237.

7. G. D. Moore, JHEP 11, 021 (2001) hep-ph/0109206.

8. M. Sallé, J. Smit and J. C. Vink, Phys. Rev. D64, 025016 (2001) hep-ph/0012346.

9. G. Aarts and J. Smit, Phys. Rev. D61, 025002 (2000) hep-ph/9906538.

10. J.-I. Skullerud, J. Smit and A. Tranberg, JHEP 08, 045 (2003) hep-ph/0307094.

11. A. Krasnitz and R. Venugopalan, Phys. Rev. Lett. 84, 4309 (2000) hep-ph/9909203.

12. A. Krasnitz, Y. Nara and R. Venugopalan, Nucl. Phys. A717, 268 (2003) hep-ph/0209269.

13. T. Lappi, Phys. Rev. C67, 054903 (2003) hep-ph/0303076. 